\title{
La elaboración del Diccionario de locuciones verbales para la enseñanza del español
}

\author{
Inmaculada Penadés Martínez \\ Universidad de Alcalá
}

\section{INTRODUCCIÓN}

Como es bien sabido, de un tiempo a esta parte, la publicación de diccionarios de orientación escolar en lengua española ha experimentado un desarrollo difícilmente imaginable hace tan sólo unos años. Estas obras lexicográficas se han elaborado teniendo como punto de referencia ya sea la enseñanza del español como lengua materna, como segunda lengua o como lengua extranjera y abarcan una tipología variada de diccionarios: desde generales de lengua hasta específicos, como es el caso de los que recogen algún tipo de unidad fraseológica.

Dos de las especialidades mencionadas, la enseñanza de español como lengua extranjera y la fraseología, vienen siendo para mí objeto de especial interés desde hace algunos años y han encontrado nuevamente su conjunción en la obra (I. Penadés Martínez, 2002) cuya elaboración voy a explicar aquí, pues, dado el estado actual de la lexicografía española, ya resulta casi impensable confeccionar un diccionario sin dar cuenta de los criterios seguidos y sin justificar las decisiones que han llevado a redactarlo de la forma en que se ha hecho' ${ }^{1}$. Esta

1 Si bien el Diccionario de locuciones verbales para la enseñanza del español va precedido de una "Presentación" y de unas "Instrucciones para el uso del diccionario", estos apartados están concebidos como una guía práctica para el usuario y, en consecuencia, no son los adecuados para iniciar desde ellos una discusión científica sobre lexicografía y fraseografía, la cual puede tener como foro más adecuado éste: un artículo en una revista especializada. 
obligación es mayor, si cabe, cuando la obra lexicográfica en cuestión vincula, como es el caso, la fraseología y la fraseografía, por el secular retraso existente en el cultivo de estas disciplinas en relación con la lengua española.

Presentado el objetivo general de este artículo, sólo resta puntualizar que la estructuración que se le ha proporcionado reproduce los pasos habituales en la confección de un diccionario. Así, el primer apartado está dedicado a explicar la selección de las locuciones verbales que registra el DICLOCVER; el segundo, a comentar su macroestructura, y el tercero, su microestructura, teniendo en cuenta que este último está dividido en tantos subapartados como partes tiene una entrada del DICLOCVER: lema, categoría, acepción, nivel, marcas, combinatoria sintagmática, definición, ejemplos, relaciones de sinonimia y antonimia e indicaciones gramaticales y pragmáticas.

\section{LA SELECCIÓN DE LAS LOCUCIONES VERBALES}

El DICLOCVER reúne un solo tipo de unidad fraseológica: locuciones propias del español hablado en esta orilla del Atlántico, más en concreto, locuciones verbales, es decir, las que equivalen a un verbo o a un sintagma verbal ${ }^{2}$. Además, constituye el primer volumen aparecido de un conjunto de volúmenes que posteriormente irán surgiendo y que registrarán locuciones correspondientes al resto de clases: adverbiales, adjetivas, sustantivas y pronominales, con excepción de las prepositivas y las conjuntivas. En principio, es un diccionario para la enseñanza del español a estudiantes de E/LE, si bien haciendo caso omiso de cierta información que, de manera específica, está dirigida a ellos y a los profesores que les imparten docencia, también puede ser utilizado

2 Valga como definición de locución la proporcionada por J. Casares (1969: 170): "combinación estable de dos o más términos, que funciona como elemento oracional y cuyo sentido unitario consabido no se justifica, sin más, como una suma del significado normal de los componentes". A partir de ahí, cabe entender que las locuciones verbales son aquellas que funcionan como lo haría un verbo en la oración o un sintagma cuyo núcleo fuera un verbo: untar la mano o vender la burra. 
en el ámbito de la enseñanza del español como lengua materna y como segunda lengua, así como por los usuarios habituales de diccionarios.

La selección de las 1942 locuciones verbales incluidas en el diccionario se ha llevado a cabo de dos modos: mediante el vaciado de una serie de diccionarios y a partir de la extracción de las locuciones verbales existentes en un conjunto de textos, correspondientes al registro escrito y oral del español, que más adelante se detallará. Los diccionarios que han constituido la base para la elaboración de éste son el Diccionario didáctico de español, intermedio (DDEI), de SM; el Diccionario para la enseñanza de la lengua española (DIPELE), de Vox; el Diccionario Salamanca de la lengua española (DSLE), y el Diccionario fraseológico del español moderno (DFEM), de F. Varela y H. Kubarth. Tal como se observa, los tres primeros son diccionarios generales de lengua, mientras que el último incluye locuciones, complejos fraseológicos con casillas vacías y expresiones, si bien sólo las primeras fueron extraídas para el DICLOCVER. Por otra parte, conviene señalar que los tres diccionarios generales y el fraseológico están orientados a usuarios que aprenden español bien sea como lengua materna o como lengua extranjera. La orientación didáctica de estas obras lexicográficas determinó su elección para conformar el conjunto de locuciones verbales que debían incluirse en el DICLOCVER. Además, el criterio de selección adoptado fue sometido a una restricción ulterior, pues todas las locuciones verbales obtenidas en el vaciado de esos diccionarios fueron examinadas a la luz de los datos proporcionados por el Diccionario del español actual (DEA) de M. Seco, O. Andrés y G. Ramos. La decisión de escoger esta obra, como punto de referencia y de contraste para incluir o no una determinada unidad en el DICLOCVER, ha estado determinada por sus propias características: el $D E A$ está redactado a partir de una base documental constituida por más de 1600 libros e impresos varios y por muchos miles de números de más de 300 publicaciones periódicas, todos ellos comprendidos entre 1955 y 1993, con lo que se garantiza la actualidad del léxico incluido (M. Seco, O. Andrés y G. Ramos, 1999: XIII-XIV). La decisión de tomar el DEA como referencia no se mostró en absoluto vana, sino todo lo contrario, pues muy pronto se pudo comprobar que los diccionarios usados como base para 
la redacción del DICLOCVER incluían como locuciones verbales o bien unidades que el $D E A$ no registraba, a pesar de su base documental, o bien unidades que no podían considerarse locuciones verbales, por ser unidades léxicas simples con régimen preposicional, colocaciones en las que uno de los elementos constitutivos es un verbo o locuciones pertenecientes a clases distintas a la de las verbales ${ }^{3}$. Todas las unidades de uno u otro grupo han sido sistemáticamente excluidas del DICLOCVER. Pero existe una excepción, se trata de unidades como apañárselas, jurársela o montárselo; su peculiar constitución con un elemento pronominal que carece de referente y su escritura en las formas personales como si se tratara de una combinación de varias palabras permiten asignar a estos elementos una posición periférica entre las unidades léxicas simples y las combinaciones fijas; junto a ello, esas mismas particularidades pueden inducir a pensar a un estudiante de español como lengua extranjera que su tratamiento lexicográfico es el propio de una unidad fraseológica y no el de una unidad simple.

Los textos escritos de los que se han tomado las locuciones verbales existentes en ellos pertenecen a dos grupos distintos: por una parte, trece obras de la colección "El Papagayo" de la Editorial Temas de Hoy ${ }^{4}$ y, por otra, cinco libros sobre Manolito Gafotas de la escritora Elvira Lindo ${ }^{5}$. El hecho de que el primer conjunto de textos, bien que li-

3 A título de ejemplo, vid. I. Penadés Martínez (2001), donde he demostrado que de 104 locuciones verbales, tomadas del DFEM, 86 pueden considerarse colocaciones a partir de las informaciones suministradas por el $D E A$.

4 En concreto, las siguientes: Coll, José Luis (septiembre 1992): El eroticoll. Diccionario erótico, Madrid, Ediciones Temas de Hoy, $5^{\text {a }}$. ed. [octubre 1991]; García Casti1lo, Julio (julio 1992): Cómo convertirse en un genio de los negocios, Madrid, Ediciones Temas de Hoy, 6a . ed. [noviembre 1989]; Gomaespuma (septiembre 1993): Familia no hay más que una y el perro lo encontramos en la calle, Madrid, Ediciones Temas de Hoy, $16^{\text {a }}$. ed. [octubre 1990]; Goméz Rufo, Antonio (junio 1993): Cómo ligar con esa chica que tanto te gusta y a la que le gusta otro, Madrid, Ediciones Temas de Hoy, 10a. ed. [abril 1989]; Malo de Molina, Carlos A. y Pérez Henares, Antonio (septiembre 1992): Cómo ser infiel sin que te descubran, Madrid, Ediciones Temas de Hoy, $8^{\text {a }}$. ed. [junio 1990]; Marqués, Josep-Vicent (junio 1992): Curso elemental de varones sensibles y machistas recuperables, Madrid, Ediciones Temas de Hoy, $8^{\text {a }}$. ed. [febrero 1991]; Pino, Marina (junio 1993): Como montártelo por el mo- 
terarios, es decir, aunque sean textos de creación, refleje el registro coloquial y de que el segundo corresponda a lo que se denomina literatura juvenil determinó que se seleccionaran para constituir el corpus de locuciones del DICLOCVER.

Por su parte, los textos orales revisados para la extracción de locuciones verbales son de distinta procedencia y tienen diferentes características. En primer lugar, se han examinado 18 conversaciones recogidas en grabaciones realizadas entre febrero y septiembre de 1991 en la ciudad de Alcalá de Henares ${ }^{6}$. Cada grabación dura entre 45 minutos y 1 hora aproximadamente, lo que supone unas 15 horas de grabación, que transcritas alcanzan un tamaño cercano a 750.000 bites y de algo más de 175.000 palabras 7 . En segundo lugar, se han escuchado, con el fin de obtener, asimismo, las locuciones verbales que hubiera en ellas,

rro. Manual del buscavidas, Madrid, Ediciones Temas de Hoy, $8^{\text {a }}$. ed. [marzo 1990]; Rico-Godoy, Carmen (abril 1994): Cómo ser infeliz y disfrutarlo, Madrid, Ediciones Temas de Hoy, 12a . ed. [noviembre 1991]; Rico-Godoy, Carmen (enero 1995): Cómo ser mujer y no morir en el intento, Madrid, Ediciones Temas de Hoy, 52 $2^{\mathrm{a}}$. ed. [mayo 1990]; Salcedo, componente de Martes y Trece (noviembre 1992): Sufro "bucho", Madrid, Ediciones Temas de Hoy, 10a. ed. [diciembre 1991]; Tola, Fernando G. (enero 1992): Cómo hacer absolutamente infeliz a un hombre, Madrid, Ediciones Temas de Hoy, 21ª . ed. [noviembre 1988]; Valls, María Antonia (junio 1992): Para quésirve un marido, Madrid, Ediciones Temas de Hoy, $5^{\text {a }}$. ed. [marzo 1992], y Zarraluqui, Luis (enero 1993): Batallas conyugales. Adulterios, traiciones, divorcios y demás contiendas matrimoniales, Madrid, Ediciones Temas de Hoy, $7^{\text {a }}$. ed. [mayo 1988].

5 Concretamente, Lindo, Elvira (1995): Pobre Manolito, Barcelona, Círculo de Lectores; Lindo, Elvira (1996): ;Cómo molo! (Otra de Manolito Gafotas), Madrid, Santillana; Lindo, Elvira (junio 1997): Los trapos sucios. Manolito Gafotas, Madrid, Santillana, 2a . ed. [1997]; Lindo, Elvira (octubre 2000): Manolito "on the road", Madrid, Santillana, 10a. ed. [1998], y Lindo, Elvira (mayo 2000): Manolito Gafotas. Yo y el Imbécil, Madrid, Santillana, 5a. ed. [1999].

6 El corpus constituyó la base para una investigación sobre alternancia de turnos en lengua española realizada por A. $\mathrm{M}^{\mathrm{a}}$. Cestero Mancera. Los resultados, así como la descripción completa de las características del corpus, pueden consultarse en A. Ma Cestero Mancera (2000). Por otra parte, el corpus fue cedido a la Real Academia Española para que formara parte de los materiales de lengua hablada del Corpus de Referencia del Español Actual (CREA).

7 Las características de estas conversaciones, en tanto que reflejo del registro coloquial, están explicadas en I. Penadés Martínez (en prensa a). 
15 conversaciones espontáneas y 9 entrevistas, que ocupan en total unas 18 horas de grabación aproximadamente. Las grabaciones de unas y otras se llevaron a cabo entre marzo y octubre de 1992. Las conversaciones se produjeron entre hablantes de una red social de Alcalá de Henares constituida por 24 informantes de distintas edades y de los dos sexos. Las entrevistas las llevó a cabo un explorador externo, y estaban dirigidas a los miembros de la red; se trata de entrevistas poco estructuradas en las que la conversación se dirigió para obtener determinada información ${ }^{8}$. En tercer lugar, se han analizado, con la finalidad anterior, 32 entrevistas semidirigidas realizadas entre enero y julio de 1994 en cuatro localidades del nordeste de la Comunidad de Madrid'. El total de horas grabadas es de 16. En cuarto lugar, del corpus de conversaciones coloquiales recogido en Briz (1995) se han vaciado, asimismo, las locuciones verbales existentes. Este corpus está compuesto por nueve conversaciones que ocupan en total 3 horas y media de grabación ${ }^{10}$. Por último, se han extraído las locuciones verbales de 16 entrevistas que se recogieron entre mayo y septiembre de 1998, como resultado de la interacción entre un investigador que participó como interlocutor y un informante que varió de una conversación a otra y sobre el que se efectuó el control de las variables sexo y edad ${ }^{11}$. Todas las conversaciones tienen no menos de 45 minutos de duración

8 Sobre las conversaciones y las entrevistas se llevó a cabo un análisis fonético y morfosintáctico con el objetivo de conocer el habla actual de los habitantes autóctonos de Alcalá de Henares. Las características completas de este corpus y los resultados del estudio pueden consultarse en A. Blanco Canales (1997).

9 En concreto, las entrevistas se hicieron a informantes de distintas edades y sexos de las poblaciones de Algete, Paracuellos de Jarama, Talamanca de Jarama y Torrelaguna, y se utilizaron como corpus para la descripción dialectal del nordeste de la Comunidad de Madrid. Los resultados de la investigación pueden consultarse en A. M. Ruiz Martínez (1999) y A. M. Ruiz Martínez (en prensa).

10 Las características de este corpus están explicadas en A. Briz (1995: 11-48).

11 El corpus forma parte de los materiales recogidos para el análisis de la lengua hablada en Alcalá de Henares, dentro del Proyecto para el Estudio del Español de España y de América (PRESEEA). Para una presentación del PRESEEA en esta ciudad véase F. Moreno Fernández, A. M ${ }^{a}$. Cestero Mancera, I. Molina Martos y F. Paredes García (2000) y F. Moreno Fernández, A. Mª . Cestero Mancera, I. Molina Martos y F. Pare- 
-lo que supone unas 12 horas y unas 147.000 palabras-, son semidirigidas y se grabaron con el magnetófono a la vista ${ }^{12}$.

Así pues, el vaciado de todos estos materiales: cuatro diccionarios, dieciocho textos escritos de creación y aproximadamente 65 horas de grabación de textos orales, y el análisis de las unidades obtenidas a partir del DEA proporcionaron las 1942 locuciones verbales que el DICLOCVER ofrece como apropiadas para la enseñanza del español.

\section{LA MACROESTRUCTURA DEL DICCIONARIO}

El DICLOCVER está formado por dos partes claramente diferenciadas: el cuerpo del diccionario propiamente dicho y los apéndices. En cuanto al diccionario, incluye las locuciones verbales ordenadas alfabéticamente por la primera palabra que constituye cada locución; cuando la primera palabra de dos locuciones es la misma, la segunda palabra determina entonces la ordenación alfabética y, así, sucesivamente. Se ha considerado que para un estudiante de E/LE, y aun para un estudiante que tiene esta lengua como materna o como segunda, es más adecuada la ordenación alfabética que la habitual en los diccionarios generales de lengua y en muchos diccionarios de unidades fraseológicas, que siguen el criterio de incluir una determinada unidad fraseológica bajo la palabra que se considera clave en el fraseologismo, apoyándose en un orden jerárquico que puede variar de un diccionario a otro, pero que, en general, se atiene a la idea de que el primer sustantivo de una locución, por ejemplo, es la palabra clave en cuya entrada hay que localizar la locución en cuestión; en caso de que no exista un sustantivo, la palabra clave puede ser un adjetivo, y, si la locución no tiene elementos pertenecientes a esta clase de palabras, la clave para buscarla puede ser el verbo o el adverbio o el pro-

des García (2002). Esta última publicación recoge también la transcripción de las entrevistas correspondientes a los hablantes de instrucción superior.

12 El carácter coloquial de las entrevistas está explicado, asimismo, en I. Penadés Martínez (en prensa a). 
nombre que la constituya ${ }^{13}$. Para un estudiante extranjero de español, que puede desconocer hasta lo que es sustantivo en su propia lengua, carece de sentido una ordenación que le obligue a llevar a cabo una reflexión metalingüística para la que puede no estar capacitado, con el fin de localizar en un diccionario una unidad fraseológica cuyo significado asimismo ignora. Algo semejante puede ocurrir en el caso de los estudiantes de español como lengua materna, si todavía no han superado la fase de aprendizaje de la clasificación de las palabras.

Los nueve apéndices que constituyen la segunda parte en la macroestructura del DICLOCVER se han confeccionado pensando en los profesores que se dedican, precisamente, a impartir docencia sobre esta lengua a extranjeros. Con la información ofrecida en ellos y con la disposición que presentan, los profesores podrán encontrar fácilmente locuciones con unas determinadas características con la finalidad de preparar material didáctico para trabajar las locuciones verbales en el aula. Los apéndices se han dispuesto en relación con los distintos apartados que conforman una entrada del diccionario. Así, en primer lugar aparecen los que recogen las locuciones del nivel intermedio (i), las locuciones del nivel avanzado (a) y las locuciones del nivel superior (s), ordenadas alfabéticamente. Se ha considerado que las locuciones marcadas con (i) son las que deben trabajarse en un nivel intermedio de enseñanza de E/LE. Todas ellas corresponden a locuciones que, estando registradas en el $D D E I$, en el DIPELE y en los textos escritos de la base documental, se encuentran, asimismo, en los textos orales escuchados para redactar el DICLOCVER. El criterio seguido para adjudicar ese nivel a las locuciones que lo presentan ha sido, pues, su uso en conversaciones reales entre hablantes de español ${ }^{14}$. El nivel (a) caracteriza las locuciones

13 Por regla general, aunque siempre se pueden encontrar excepciones, estos criterios son explicados en las partes introductorias de los diccionarios.

14 Cuestión distinta es la frecuencia de uso de estas locuciones en las conversaciones y entrevistas examinadas, aspecto que por ahora no se ha tenido en cuenta. El análisis de esta cuestión es extremadamente complejo por la necesidad de poner en relación 
verbales que podrían corresponder a una enseñanza avanzada y se ha asignado a las locuciones registradas en el DDEI y en el DIPELE, en los textos escritos utilizados (con la excepción de las locuciones que, además de encontrarse en todas estas obras, también aparecen en los textos orales, pues, en ese caso, su nivel es el (i), tal como se acaba de explicar), en el DSLE y el DFEM, pero en estos dos últimos casos siempre que, a su vez, estuvieran presentes, asimismo, en los textos orales examinados. El hecho de que el DDEIy el DIPELE estén dirigidos a escolares comprendidos entre los doce y los dieciséis años que aprenden español como primera lengua -aunque el segundo también a las personas que aprenden esta lengua como extranjera- y de que recojan un menor número de entradas que el DSLE y que el $D F E M$ ha determinado que se decidiera asignar las locuciones verbales existentes en ellos a un nivel avanzado de enseñanza de español. Por otra parte, también ha parecido conveniente marcar con ese mismo nivel las locuciones extraídas de los dos tipos de textos escritos vaciados, en el caso de los textos de la colección "El Papagayo" porque reflejan en gran medida el registro coloquial del español y, en el caso de las obras de literatura juvenil, porque van dirigidas a un público semejante a los usuarios de los dos diccionarios anteriores. Por último, hay que entender que las locuciones marcadas con (s) deben trabajarse en un nivel superior de enseñanza de E/LE. Las que presentan esta marca son locuciones verbales que registran única y exclusivamente el DSLE y el DFEM. Tanto uno como otro cuentan con un mayor número de entradas que los otros dos diccionarios, de ahí que las locuciones que sólo han aparecido registradas en ellos podrían constituir el conjunto idóneo para la enseñanza del nivel superior. Habrá podido observarse que en la propuesta de enseñanza de las locuciones verbales, presentada aquí de manera implícita, se entiende que ésta debe iniciarse en el nivel intermedio y no en el elemental, pues parece más conveniente dedicar éste al aprendizaje de otros

el uso de una locución con el uso del verbo simple o del sintagma verbal libre al que equivale, con el uso de cualquier verbo simple y, asimismo, con el uso de otras locuciones verbales. Algunos datos en relación con esta problemática pueden encontrarse en I. Penadés Martínez (en prensa a). 
contenidos lingüísticos, dada la mayor complejidad de estas unidades fraseológicas ${ }^{15}$.

Pese a las justificaciones ofrecidas para determinar el nivel de cada locución verbal del DICLOCVER, podría creerse que las decisiones adoptadas son de todo punto arbitrarias. Y tal vez así pudiera ser. Pero no hay que olvidar que la enseñanza de las unidades fraseológicas en $\mathrm{E} / \mathrm{LE}$ cuenta todavía con muchas lagunas; una de ellas es, precisamente, el desconocimiento de qué locuciones, por ejemplo, hay que enseñar en función del nivel en el que se encuentra el alumno. De algún modo hay que iniciar el camino que conduzca a la obtención de ese tipo de datos, y, en este sentido, el DICLOCVER ofrece una primera propuesta, sujeta, claro está, a las modificaciones que se demuestren oportunas y convenientes.

Después de los apéndices que distribuyen las locuciones verbales en niveles, figuran los que recogen, siguiendo también el orden alfabético, las "Locuciones con la marca infor.", las "Locuciones con la marca vulg." y las "Locuciones sin marca de registro". Las marcas infor. y vulg. se han asignado a partir de las indicaciones de los diccionarios que se han consultado para la confección de éste ${ }^{16}$. Se considera que las locuciones que cuentan con la abreviatura infor. se usan en situaciones informales, familiares o de confianza. Por su parte, la abreviatura vulg. informa acerca de que el uso de las locuciones verbales que la presentan es disfemístico, por lo que no resulta adecuado utilizarlas en público ni en situaciones en las que exista poca confianza entre los interlocutores. Otras muchas locuciones, recogidas también en el apéndice correspondiente, carecen de marca. En este caso, hay que entender que la mayoría de ellas corresponde a un uso normal o estándar y sólo unas

15 La idea es compartida por otros autores; vid., en este sentido, M.M. Forment Fernández (1998: 339). Por otra parte, para un mayor desarrollo de estas cuestiones vid. I. Penadés Martínez (1999).

16 En concreto, además del DDEI, el DIPELE, el DSLE, el DFEM y el DEA, los de la Real Academia Española (1992) y (2001) - esta última edición no desde que se inició la confección del DICLOCVER, pues no se había publicado todavía, sino desde su aparición-y el de María Moliner (1966-1967). 
pocas, a un registro formal, que no se ha marcado de momento, dada la situación en que se encuentran los estudios sobre variación diafásica en fraseología y la consiguiente dificultad a la hora de ofrecer este tipo de información.

Las definiciones de las locuciones permiten agrupar muchas de ellas por lo que he denominado campos conceptuales, es decir, se pueden reunir dentro de un mismo campo conceptual las que expresan una determinada idea o concepto ${ }^{17}$; por esta razón, se ha elaborado un apéndice de "Locuciones registradas por campos conceptuales"18. La confección de estos 28 campos, que incluyen 1299 locuciones (lo que representa el $66,8 \%$ del total de locuciones del diccionario) me ha supuesto enfrentarme a muchos problemas, de cuya resolución no estoy plenamente satisfecha. Estoy convencida, además, de que esas mismas dudas asaltarán a todo aquel que lo maneje y de que las disensiones con mi propuesta no serán pocas. Considero, no obstante, que, tal y co-

17 Puede que el término campo conceptual recuerde el uso que de él hizo el lingüista $\mathrm{O}$. Ducháček; sin embargo, en la práctica no se ha procedido como este autor, que excluyó de su estudio del campo conceptual de la belleza en francés moderno la mitad del campo: las palabras relativas a la fealdad, como muy bien señaló E. Coseriu (1964: 155). Así pues, los campos conceptuales del DICLOCVER incluyen locuciones que, expresando un determinado concepto: movimiento, sexo, hablar, etc., pueden presentar entre sí o no relaciones de sinonimia, de antonimia y de cohiponimia.

18 Los campos son los siguientes: 1) 'acciones, estados y procesos propios de una persona como ser vivo'; 2) 'acciones realizadas diciendo determinadas palabras a una persona'; 3) 'acciones referidas al movimiento'; 4) 'acciones y procesos referidos al sexo'; 5) 'actividad intelectual'; 6) 'actividad profesional'; 7) 'actuar una persona'; 8) 'atribución de cualidades a una cosa'; 9) 'atribución de cualidades a una persona'; 10) 'causar daño a una cosa o sufrirlo'; 11) 'causar daño físico a una persona o sufrirlo'; 12) 'causar un estado de ánimo o un sentimiento en una persona'; 13) 'comportarse una persona de una determinada manera'; 14) 'conocimiento'; 15) 'consecución u obtención de una cosa'; 16) 'creencias u opiniones'; 17) 'dar a conocer, manifestar o mostrar'; 18) 'decir y hablar'; 19) 'encontrarse una persona o una cosa en una situación'; 20) 'estar una persona de una determinada manera'; 21) 'existencia de una cosa'; 22) 'experimentar un estado de ánimo o un sentimiento'; 23) 'fingimiento'; 24) 'ocurrir o suceder una cosa'; 25) 'percepción física e intelectual'; 26) 'posesión no material'; 27) 'realización de una cosa', y 28) 'rechazar o despreciar a una persona o una cosa'. 
mo se presenta, el apéndice ofrece muchas posibilidades para trabajar las locuciones verbales en el aula, no de manera aislada o sin establecer entre ellas más que relaciones puramente fortuitas o anecdóticas, sino vinculándolas por su significado, por su combinatoria sintagmática y por su significante.

La misma utilidad tiene el apéndice de "Locuciones sinónimas y antónimas", donde se registran todas las series de locuciones sinónimas y de locuciones sinónimas y antónimas a que se hace referencia en el cuerpo del diccionario, pues mediante su consulta se pueden obtener conjuntos de unidades fraseológicas entre las que existen las relaciones semánticas de sinonimia y antonimia. Estas relaciones de significado se han establecido como resultado del examen de las definiciones de las locuciones proporcionadas por todos los diccionarios consultados para la redacción del DICLOCVER y se reflejan, asimismo, en las propias definiciones del DICLOCVER. El apéndice ofrece material adecuado para la elaboración de ejercicios sobre locuciones verbales basados en su identidad u oposición de significado ${ }^{19}$.

Que los profesores de E/LE tuvieran recogidas de manera conjunta las series de locuciones sinónimas y antónimas que se han obtenido como resultado de la confección del diccionario no fue la única razón para preparar este apéndice. También pesó el hecho de que señalar dentro de una entrada todas sus locuciones sinónimas hubiera complicado en exceso su presentación formal, además de que la extensión del diccionario se hubiera acrecentado sobremanera, pues una misma serie de sinónimos, por ejemplo, se hubiera encontrado

19 No son estas dos las únicas relaciones semánticas que pueden establecerse entre estas unidades fraseológicas. Vid., en este sentido, I. Penadés Martínez (2000), y, aunque no se ofrece en el DICLOCVER un apéndice de locuciones hipónimas, a partir de las definiciones proporcionadas en él puede deducirse la relación de hiponimiahiperonimia existente entre muchas locuciones del DICLOCVER y un lexema simple de la lengua. Piénsese, por ejemplo, que locuciones del campo conceptual relativo a 'decir y hablar' son hipónimas de las unidades léxicas simples del español hablar o decir: alzar la voz, coger por banda', dar a entender, dar cuerda, decir lo que (se) le viene a la boca, decir para su capote, hablar como un libro (abierto), hablar por boca, hablar por boca de ganso, hablar por hablar, etc. 
repetida en todas y cada una de las entradas relativas a las locuciones que la constituyen.

Por todo ello, si una locución tiene solamente otra como sinónima o como antónima se remite a ella en la propia entrada, tal como en el siguiente apartado del artículo se explicará. Pero cuando una locución tiene más de una sinónima, es decir, forma parte de una serie sinonímica en la que, además, las distintas locuciones sinónimas pueden tener una o varias antónimas, en la entrada correspondiente a la primera locución de la serie, según el orden alfabético, aparece su lema precedido de un asterisco $(*)$ en negrita:

caer bien v. (i) [alguien, a alguien] Resultar simpático \{a una persona\}: Es una mujer muy agradable y a mí me cae bien. *caer bien.

La indicación se repite en todas las entradas que constituyen la serie:

caer gordo v. (a) infor. [alguien, a alguien] Resultar antipático \{a una persona\}: No le digas que venga con nosotros, a mi me cae gordo. *aer bien

caer mal v. (i) infor. [alguien, a alguien] Resultar antipático \{a una persona\}: No me explico cómo puedes ser su novia, a todo el mundo le cae mal; Han declarado que lo apuñalaron a la puerta de la discoteca porque les cayó mal. *caer bien

no ser santo de la/su devoción v. (s) infor. [alguien, de alguien] Resultar antipático a una persona: El primer ministro no es santo de la devoción ni de los miembros de la derecha ni de los de la izquierda de su propio partido. *caer bien,

con el fin de remitir al lector al apéndice de "Locuciones sinónimas y antónimas", donde encontrará todas las series, ordenadas alfabéticamente por su primera locución. A su vez, en el apéndice, dentro de cada serie se han dispuesto, bajo la locución que funciona como cabeza de la serie, todas las sinónimas ordenadas, asimismo, alfabéticamente:

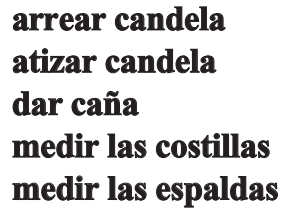




\section{meter caña ${ }^{1}$ \\ poner la mano encima \\ sacudir el polvo \\ zumbar la pandereta \\ zurrar la badana.}

En el caso de existir también antónimas, éstas se diferencian de las sinónimas por una línea de separación:

\section{caer bien \\ caer gordo \\ caer mal}

no ser santo de la/su devoción.

Por último, y dado que, habitualmente, las locuciones se incluyen en los diccionarios bajo la entrada correspondiente al primer sustantivo que forma una locución, se ha confeccionado un apéndice de "Locuciones registradas bajo los sustantivos que las constituyen". Puede que, tomado en su conjunto, resulte menos práctico que los otros, pero, al menos, sirve para mostrar la gran rentabilidad de los sustantivos que designan diferentes partes del cuerpo humano para formar muchas locuciones, las llamadas somatismos, y también para vincular, en muchos casos, el significado de una locución verbal con el significado de los elementos que la forman, pues no en balde existe lo que se denomina motivación fraseológica.

\section{LA MICROESTRUCTURA DEL DICCIONARIO}

Cada entrada del DICLOCVER proporciona informaciones, sobre una serie de aspectos de las locuciones verbales, que se han organizado en los apartados habituales de un artículo de diccionario, tal como a continuación se mostrará.

\subsection{EL LEMA}

El lema de la locución verbal de la que se ocupa un artículo está señalado en negrita. A veces, parte del lema de una locución aparece entre paréntesis: haber oído campanas (y no saber dónde). Se indica con ello que la locución puede usarse bajo la forma que incluye la 
parte situada entre paréntesis (haber oído campanas y no saber dónde) o bajo la forma que no la contiene (haber oído campanas). Para la ordenación alfabética de la locución, se considera su forma completa: haber oído campanas y no saber dónde. En otras ocasiones, el lema de una locución verbal contiene una barra (/) que separa dos o más palabras o dos partes de la locución: echar su/un cuarto a espadas o no llegar (ni) a la suela de los zapatos / del zapato. La barra se utiliza para indicar que la locución tiene dos formas: una constituida con el elemento situado a la izquierda de la barra (echar su cuarto a espadas y no llegar (ni) a la suela de los zapatos) y la otra, con el elemento situado a la derecha de la barra (echar un cuarto a espadas y no llegar (ni) a la suela del zapato). La ordenación alfabética de las locuciones que contienen una barra se ha llevado a cabo contando sólo con el elemento situado a su izquierda; por eso, echar su/un cuarto a espadas se ordena como si la forma fuera únicamente echar su cuarto a espadas.

Otras variaciones léxicas de una misma locución verbal han recibido un tratamiento condicionado por los usuarios a los que de manera primordial va dirigido el diccionario: los estudiantes de E/LE. De ahí que si una locución presenta dos o más posibilidades léxicas alternas para la formación de su lema, éstas no se han señalado en una misma entrada mediante el uso de la barra, sino que aparecen como entradas distintas ordenadas alfabéticamente en el lugar que les corresponde, entradas entre las que se han establecido remisiones de sinonimia, como ocurre con andar el diablo suelto y estar el diablo suelto, por ejemplo.

Por su parte, las variaciones morfológicas que no constituyen formas alternas del lema, sino que representan la opción de incluir o no en el lema determinada unidad morfológica ${ }^{20}$, no se recogen en esta parte de la entrada, sino en la de indicaciones gramaticales y pragmáticas. Es decir, la posibilidad de formar la locución prometérselas felices

20 Son casos distintos al de llamar a la/su puerta, señalado con el uso de la barra, tal como se acaba de explicar. 
bajo el lema prometérselas muy felices no se ha indicado presentando el lema con la forma prometérselas (muy) felices, como ocurre en otros diccionarios, sino mediante la observación oportuna en el apartado dedicado a informaciones gramaticales. La razón estriba en el hecho de que, en algún caso, las unidades morfológicas que de forma optativa pueden intercalarse entre los elementos constitutivos de una locución son más de una, y presentarlas todas ellas en el lema de la unidad fraseológica hubiera supuesto una complicación innecesaria, pues hubiera quedado el lema de un modo semejante al siguiente: dar (demasiada / mucha / poca) guerra. De ahí que se hayan tratado sistemáticamente estas posibilidades de variación morfológica en otra parte del artículo.

\subsection{LA CATEGORÍA}

La asignación de la categoría gramatical a una locución es un tema complejo que merece un estudio aparte ${ }^{21}$. Con todo, en el DICLOCVER se han marcado las locuciones con la abreviatura $\boldsymbol{\nu}$. (locución verbal), que señala su pertenencia a la clase de las verbales. Por estar este volumen dedicado a esta clase, todas tienen la misma marca categorial. No obstante, en algunos casos, la abreviatura utilizada ha sido $\boldsymbol{\nu}_{\text {-pprnl. }}$ (locución verbal pronominal); se indica con ella que la locución en cuestión puede usarse o no, de manera opcional, con las formas pronominales me, te, se, nos, os, se. Así,

21 Sólo a título de ejemplo, piénsese que estar al borde es, según el DDEI, una locución verbal, pero el $D E A$ la registra como locución preposicional bajo el lema al borde de; ir de boca en boca sería, asimismo, una locución verbal, según el DIPELE, para el $D E A$, en cambio, es una locución adverbial: de boca en boca; (como) cosa de debería considerarse por su paráfrasis definitoria en el DSLE una locución adverbial, en el DEA se clasifica como preposicional bajo el lema cosa de, y, por último, llover cuatro gotas debería ser clasificada como locución verbal, según el lema y la paráfrasis definitoria proporcionados por el DFEM, si atendemos, en cambio, al DEA, esa locución tiene el lema cuatro (o dos) gotas y debe clasificarse como nominal. Para la confección del DICLOCVER se ha seguido el criterio del DEA, aunque sería necesaria la realización de un estudio sobre la clasificación de las locuciones para analizar su funcionamiento a partir de ejemplos registrados en un corpus de datos. 
por ejemplo, con la locución saltar a la torera es posible construir expresiones con la forma pronominal o sin ella: Si te saltas a la torera la prohibición de adelantar, algún día tendrás un accidente o Han saltado a la torera los acuerdos firmados.

Junto a este tipo de locución verbal, que opcionalmente admite la aparición o no de la forma pronominal, sin que ello suponga un cambio de significado, existen otras locuciones en las que la aparición del se conlleva para la locución una combinatoria sintagmática distinta y una variación, no en el significado léxico, sino en el diatético. Conviene tener en cuenta que, en estos casos, la locución se ha ordenado en la macroestructura del diccionario considerando que la presencia del se es obligatoria para su combinatoria sintagmática y para su significado, que el lema siempre se construye con el se y que no hace falta marcar la locución como $\boldsymbol{\nu}_{\mathbf{0}}$-prnl., porque el propio lema ya lo indica. Corresponden a estas características locuciones como alterarse los nervios ('ponerse muy nervioso'), diatésis distinta a la de la locución alterar los nervios ('poner a una persona muy nerviosa'), o revolverse el estómago ('sentir repugnancia'), diatésis diferente a la de revolver el estómago ('causar repugnancia a una persona').

\subsection{LA ACEPCIÓN}

Hay casos en que una entrada del DICLOCVER corresponde a una locución que tiene una sola acepción y casos en que una entrada incluye todas las acepciones de locuciones con lema idéntico. Si se da esto último, el lema y la categoría de las locuciones homónimas no se repiten, pero cada acepción va encabezada por un número arábigo $(\mathbf{1}, \mathbf{2}, \mathbf{3})$ en negrita, seguido del nivel de enseñanza de la locución y de la marca de registro -cuando la acepción la tiene-, pues es posible que estas informaciones no coincidan para todas las acepciones:

cortarse la coleta v. 1 (a) [alguien] Abandonar una afición o una profesión: Tengo vocación de actor desde niño y pienso seguir así, aunque yo me quería cortar la coleta a los treinta años. 2 (s) [alguien] Retirarse de la práctica del toreo profesional: El torero se ve ya mayor y dice que, cuando acabe la temporada, se corta la coleta. 
Un aspecto que suele tenerse en cuenta a la hora de confeccionar un diccionario es el relativo a la ordenación de las acepciones en una entrada, ordenación que se lleva a cabo en unas ocasiones en función de criterios etimológicos y, en otras, de acuerdo con el criterio de acepción común o usual / acepción restringida. En el caso del DICLOCVER, no se ha procedido a un examen de las acepciones para ordenarlas, posteriormente, de acuerdo con uno u otro criterio, y ello porque, para hacer esa ordenación, hubiera sido necesario disponer tanto de estudios etimológicos sobre todas y cada una de las locuciones incluidas, como de análisis sobre su frecuencia de uso. Dado que carecemos de tales datos, la ordenación de las acepciones en este diccionario no sigue, explícitamente, ninguno de esos criterios y corresponde a la que ofrecen los diccionarios tomados como base para su redacción, haciendo caso omiso de posibles diferencias existentes entre ellos.

Al referirme, en el punto anterior, a las locuciones verbales que se construyen obligatoriamente con $s e$, he indicado que su situación en la macroestructura del diccionario está determinada por la ordenación alfabética que origina la presencia obligatoria del se. Existía también la posibilidad de ordenar locuciones como guardarse las espaldas dentro de la entrada guardar las espaldas como una acepción o subacepción de la misma. Se ha preferido, sin embargo, respetar el orden alfabético por dos razones fundamentalmente: en primer lugar, porque no siempre el significado de la locución con se es una diátesis distinta al significado de la locución sin $s e^{22} \mathrm{y}$, en segundo lugar, porque no se debe obligar a un estudiante de E/LE a relacionar hacerse añicos con hacer añicos, para encontrar en el diccionario la primera de estas locuciones ${ }^{23}$, pues es muy posible que desconozca ambas y todavía más la relación diatética existente entre ellas.

22 Así, dejar caer, con el significado de 'decir una cosa como sin querer, pero con intención', no es una diátesis diferente a dejarse caer, que tiene un significado léxico completamente distinto al de la locución anterior: 'presentarse en un lugar ocasionalmente'.

23 De hecho, ambas en el DICLOCVER están separadas por 112 entradas. 


\subsection{EL NIVEL}

Como se ha señalado más arriba, el DICLOCVER asigna a cada locución o a cada acepción el nivel que en la enseñanza-aprendizaje de E/LE podría corresponderle, teniendo en cuenta que (i) se ha adjudicado al nivel intermedio, (a) al avanzado y (s) al superior. Las locuciones marcadas con (i) son en total 186; las del nivel avanzado, 1106, y las destinadas al nivel superior, 650. El interés de esta información proporcionada por el DICLOCVER aumenta si se conjuga con otras informaciones ofrecidas. Así, por ejemplo, las locuciones sinónimas pueden diferenciarse en función del nivel de enseñanza en que pueden introducirse: meter la pata (i), meter la gamba (a) y meter la pezuña (s). Ello supone, en primer lugar, un menor esfuerzo memorístico por parte del alumno a la hora de retener unas unidades cuyo significado no se corresponde con el significado de los elementos que las constituyen, pues en el nivel intermedio sólo debe aprender unas pocas, y, en segundo lugar, un apoyo mnemotécnico para el aprendizaje de otras locuciones, sinónimas de alguna o algunas que ya conoce, en niveles más elevados de enseñanza.

\subsection{LAS MARCAS}

Ya queda dicho que el DICLOCVER ofrece información sobre la variación diafásica de las locuciones verbales o de las distintas acepciones de una locución homónima. Esta información se presenta mediante las abreviaturas infor. (informal) y vulg. (vulgar) o a través de la no marcación de una locución verbal y no es fruto de un análisis de todas y cada una de las locuciones incluidas en el DICLOCVER, sino que, simplemente, reproduce los datos que sobre este aspecto proporcionan los siete diccionarios que en todo momento se han tenido presentes para la redacción de éste. El problema que se ha suscitado con esta manera de proceder ha sido la falta de coincidencia en la asignación de las marcas o en la no marcación por parte de los diccionarios que se han tomado como referencia, pues si en muchos casos la coincidencia entre todos ellos es total, en otros muchos es patente la disparidad de tratamiento otorgado a las locuciones en esta cuestión. Así, por 
ejemplo, la locución matar una mosca no tiene ninguna marca en el $D E A$, mientras que en el DFEM aparece marcada como inf. (informal) bajo el lema no haber u. p. matado/no ser u. p. capaz de matar una mosca ${ }^{24}$; por su parte, la locución exhalar el/su espiritu tiene asignado un uso elevado en el DSLE y literario en el $D E A$, pero carece de marca de registro en el DRAE. La falta de estudios sobre la variación diafásica en las unidades fraseológicas del español puede ser, entre otras razones, la causa de las disparidades en la utilización de las marcas entre unos y otros diccionarios. Y de ahí que, cuando los diccionarios examinados no presentaban las mismas marcas, se tomara para el DICLOCVER como criterio discriminador el DEA y, fundamentalmente, el DRAE.

La marca diafásica de las locuciones, puesta en relación con otros datos del DICLOCVER, por ejemplo la relación semántica de sinonimia, permite preparar ejercicios para trabajar aspectos de las locuciones relativos a sus condiciones de uso. Así, en la serie sinonímica $h a-$ cer caso omiso, pasarse por el arco de triunfo, pasarse por el forro (de los cojones), ponerse por montera y saltar a la torera, se encuentran locuciones informales (pasarse por el arco de triunfo y saltar a la torera), vulgares (pasarse por el forro (de los cojones)) y no marcadas (hacer caso omiso y ponerse por montera), lo que le facilita al profesor la ilustración del material didáctico destinado a trabajar los aspectos pragmáticos de las unidades fraseológicas.

\subsection{LA COMBINATORIA SINTAGMÁTICA}

El tratamiento lexicográfico de los elementos del contorno de una unidad fraseológica es dispar en los distintos diccionarios, dado que en unos casos aquéllos se representan a través de unidades co-

24 Este último diccionario indica sistemáticamente el ámbito de aplicación de todas las unidades fraseológicas que contiene con las abreviaturas f. (formal), inf. (informl) o rest. (restringido), para lo que parece seguir el criterio del $D R A E$, sólo que, cuando este diccionario marca una unidad fraseológica como fam. (familiar), el DFEM sustituye este término por el de inf., mientras que para los casos en que el DRAE no establece ninguna marca, el DFEM usa f. 
mo alguien y algo escritas con el mismo tipo de letra que la unidad fraseológica en cuestión; en otras ocasiones se utilizan abreviaturas del tipo u. p. (una persona) y u.c. (una cosa), también con el mismo tipo de letra que la unidad fraseológica registrada; otras veces el lema contiene tales elementos, pero en un tipo de letra distinto al de los componentes de la unidad fraseológica para distinguirlos de éstos, e incluso se utilizan los paréntesis para diferenciar los elementos del contorno de los propios de la unidad fraseológica. A primera vista, pudiera parecer que la aparición de los elementos del contorno como parte integrante del lema de una locución puede ser útil para informar así al usuario del diccionario de sus posibilidades de combinación sintagmática. Sin embargo, tal beneficio queda mermado si se tiene en cuenta que: $1^{\circ}$ el usuario de diccionario, y sobre todo cuando es un extranjero, puede pensar que los elementos del contorno son constitutivos de la locución ${ }^{25} ; 2^{\circ}$ la aparición del contorno en el lema de la unidad fraseológica puede distorsionar la presentación de este último por obligar a una concordancia que impide la reproducción de la locución en su forma canónica ${ }^{26} ; 3^{\circ}$ los elementos del contorno no se incluyen ni se excluyen de manera sistemática en el lema de todas las unidades fraseológicas, ni aun en un mismo diccionario ${ }^{27}$, y $4^{\circ}$ no existe unanimidad de criterio entre los lexicógrafos acerca de cuáles y cuántas sean las unidades

25 De hecho la locución verbal dar algo contiene un elemento, algo, que se corresponde con uno de los que usualmente se utilizan para marcar una clase de elementos del contorno. Si ahí algo es constitutivo de la locución, lo mismo puede pensar un usuario extranjero del algo de caber en la cabeza algo, pillar de nuevas algo o salir de las narices algo, por ejemplo, si no se diferencia de los otros elementos de la locución, aunque en estos últimos ejemplos algo es un elemento del contorno.

26 Así, por ejemplo, el DFEM, en contra de toda práctica lexicográfica, presenta locuciones bajo la forma femenina por incluir como parte integrante los elementos $u$. $p$. (una persona) o $u$. c. (una cosa): (ser u. p.) más boba/tonta que hecha de encargo, [estar u. p.] sola como/más sola que un hongo o [estar u. p.] (tan contenta) como/más contenta que un niño con zapatos nuevos.

27 El DIPELE, por ejemplo, no los incluye, pero hay ocasiones en que aparecen: caerse el pelo a alguien, no llegarle a una persona la camisa al cuerpo, ser el ojo derecho de alguien, etc. 
que permitan reproducir los elementos del contorno de una unidad fraseológica ${ }^{28}$.

Ante tal situación, para el DICLOCVER se decidió que el lema de las locuciones verbales quedara restringido a lo que específicamente es su forma y que en cada artículo del diccionario figurara un apartado para la información relativa a la combinatoria sintagmática de la locución, de manera análoga a como se procede en los diccionarios de lengua que señalan en un apartado destinado a ello el régimen preposicional y los tipos de complementos que llevan los verbos. De este modo, todas las acepciones que lo exigen presentan, antes de la definición, su contorno entre corchetes, [ ], es decir, se señalan, separándolos mediante comas, los elementos con los que se combina la locución en esa acepción: el sujeto, si lo tiene, y los complementos, si es el caso. Los elementos que constituyen la combinatoria de una acepción se representan, como es habitual en la práctica lexicográfica, mediante las formas: alguien (para persona), algo (para cosa), algún lugar, algún modo, etc. Se informa, asimismo, de las preposiciones que introducen los complementos: caer gordo [alguien, a alguien]. Cuando en una misma locución pueden alternar varios tipos de elementos del contorno o varias preposiciones, las distintas posibilidades se separan mediante una barra (/): dar abasto [algo/alguien, a/para algo]. Por último, conviene señalar que la asignación del contorno a cada locución se ha llevado a cabo a partir de los ejemplos de uso de esa locución que se encuentran en los diccionarios consultados y en el corpus documental manejado, por lo que no existe la completa seguridad de que el contorno ofrecido agote todas las posibilidades combinatorias de una locución.

\subsection{LA DEFINICIÓN}

Las definiciones se han redactado teniendo siempre presentes los siete diccionarios manejados para la confección del DICLOCVER.

28 Piénsese que para referirse a un animado humano se usa tanto alguien, como una persona o uno. 
Muchas de ellas siguen el modelo de definición sinonímica, si bien se ha procurado que el sinónimo usado fuera la unidad léxica simple a la que equivale la acepción y no una locución verbal sinónima de la definida: coger el portante v. (a) infor. [alguien] Marcharse. Otras definiciones corresponden a la denominada hiperonímica: vivir del cuento v. (s) infor. [alguien] Vivir sin trabajar; se trata de una manera de vivir y, en consecuencia, la locución es un hipónimo del lexema vivir. Y también hay locuciones verbales cuyo significado se presenta a través de una paráfrasis definitoria: apañárselas v. (i) infor. [alguien, de algún modo] Encontrar la manera de resolver o solucionar alguna cosa.

Cuando en una definición aparecen elementos que se corresponden exactamente con los señalados previamente como contorno de la acepción, se han situado en la definición entre llaves, \{\} : andar a la greña v. (a) infor. [alguien] Reñir \{dos o más personas $\}$, arrojar el guante v. (a) [alguien, a alguien] Desafiar \{a una persona $\}$ o darse de bofetadas v. (a) infor. [algo, con algo] Desentonar \{una cosa con otra\}. La razón para actuar de esa manera es la que apuntan M. Seco, O. Andrés y G. Ramos (1999: XXI-XXII): presentar en la fórmula definitoria esos elementos como lo que son, elementos habituales del contorno que no constituyen parte sustancial del significado de esa locución; en otras palabras, andar a la greña significa 'reñir', arrojar el guante, 'desafiar' y darse de bofetadas, 'desentonar'; el resto de la definición en los tres casos reproduce la información presentada con anterioridad sobre el contorno de esas locuciones. Sin embargo, no se ha procedido así cuando no se da una correspondencia exacta entre los elementos señalados previamente como contorno y los elementos correspondientes en la definicicón: dar carpetazo v. (s) [alguien, a algo] Desistir de una idea o de un proyecto, donde la parte de la definición 'de una idea o de un proyecto' no figura entre llaves. La razón estriba en el hecho de que colocar entre llaves en la definición elementos que no se pueden identificar exactamente con los especificados antes como contorno -porque el verbo utilizado en la paráfrasis definitoria tiene un régimen preposicional distinto al de la locución (desitir de / dar carpetazo a)hubiera podido crear confusión a los usuarios del diccionario, sobre to- 
do a los extranjeros, que hubieran podido pensar que la locución en cuestión se construye con un complemento introducido tanto por $a$ como por de.

\subsection{LOS EJEMPLOS}

La importancia de los ejemplos de uso de las locuciones en un diccionario como éste resulta innegable. Por eso todas las acepciones definidas van seguidas, al menos, de un ejemplo en cursiva; si aparecen dos, van separados por un punto y coma (;): irse a hacer gárgaras v. (a) infor. [algo] Fracasar: Yo no sali en la portada porque la revista se fue a hacer gárgaras. Los ejemplos sirven como justificación de la definición dada a la acepción y como reflejo de las informaciones que se proporcionan en otras partes de la entrada -especialmente, en la dedicada a la combinatoria sintagmática de la locución, sobre todo cuando ésta muestra varias posibilidades-, por eso, en numerosos casos, aparece más de uno:

llevarse por delante v. (i) infor. [algo/alguien, algo/a alguien] Atropellar, derribar a una persona o una cosa que se interpone en el camino \}: El autobús se llevó por delante una farola; Pensó que se la llevaban por delante, vamos que la mataban.

Muchos de ellos se han construido ad hoc, pero intentando que respondieran a un uso real; otros se han recogido de los textos escritos y de las conversaciones grabadas tomados como base documental para la confección de este diccionario, y, por último, una parte de ellos se ha obtenido de la consulta del Corpus de Referencia del Español Actual (CREA) de la Real Academia Española. En ocasiones, en los ejemplos sacados de la base documental consultada y del CREA, ha habido que proceder a modificar de algún modo la forma del ejemplo para facilitar su comprensión por el usuario del diccionario, dada la inevitable descontextualización que toda extracción supone. Posiblemente, el primer conjunto de ejemplos sea más numeroso que el resto, porque las locuciones verbales no son de uso tan frecuente como a primera vista pudiera parecer, a pesar de las afirmaciones que, en sentido contrario, se encuentran en muchos estudios sobre el español 
coloquia ${ }^{29}$. Además, y aunque pueda resultar sorprendente, hay locuciones del diccionario que no están registradas en el corpus de la Academia, pese a su extensión.

La situación descrita en el párrafo anterior es semejante a la que encontramos en otras lenguas. En efecto, para el caso del inglés, R. Moon (1997: 50) señala el dato de que J. Strässler encontró sólo 92 ejemplos de locuciones $\mathrm{s}^{30}$ en un corpus de alrededor de 100.000 palabras formado por interacciones habladas de distintos tipos. La propia R. Moon (1997: 52) en investigaciones llevadas a cabo sobre el corpus COBUILD con 4.000 locuciones ha comprobado que un $30 \%$ aparece menos de una vez por diez millones de palabras; el 35\% aparece entre una y tres veces por diez millones de palabras, y el 20\% tiene una frecuencia de al menos un ejemplo por dos millones de palabras. Esta investigadora concluye (R. Moon 1997: 52-54) que hay muchas unidades pluriverbales en la lengua, pero una parte de ellas es muy poco frecuente; asimismo, afirma que las locuciones están especialmente asociadas tanto al género del periodismo, como al de la conversación informal. Ahora bien, cabría precisar que hay varios tipos de locuciones $y$, en lo que respecta a las verbales, los datos extraídos del corpus examinado en I. Penadés Martínez (en prensa a) -que es un parte del consultado para la redacción del DICLOCVER- no parecen mostrar una elevada frecuencia de uso en el género de la conversación informal.

\subsection{LAS RELACIONES DE SINONIMIA Y ANTONIMIA}

Corpas Pastor (1996: 50-52) separa la clase de las locuciones de las otras clases de unidades fraseológicas por su pertenencia al sistema de la lengua. Una afirmación de esta naturaleza sólo encuentra su confirmación si se muestran las relaciones semánticas existentes en-

29 De hecho, en las aproximadamente 65 horas de grabación escuchadas sólo hay registradas alrededor de 200 locuciones verbales distintas, de las 1942 incluidas en el DICLOCVER, aunque algunas de ellas aparecen con una alta frecuencia, como darse cuenta o hacer falta, frente a otras de las que sólo se ha registrado una ocurrencia, como hacer el vacio o hincar los codos.

30 Traduzco por locución el término inglés idiom. 
tre las locuciones, pues los elementos del sistema se caracterizan, frente a los de la norma y el habla, por las relaciones funcionales que establecen entre sí. En otros trabajos (I. Penadés Martínez (2000) e I. Penadés Martínez (en prensa b)) he analizado la relación semántica de hiponimia-hiperonimia entre locuciones y lexemas de la lengua y la relación de antonimia entre locuciones. En efecto, en la primera de las obras citadas, he demostrado, a partir de una revisión crítica de las concepciones sobre la relación semántica de hiponimia-hiperonimia de J. Lyons (1993) y G. Kleiber e I. Tamba (1990), que locuciones como alzar la voz ('hablarle a alguien con insolencia o faltándole al respeto', según el DFEM, p. 292), darle a la lengua ('hablar mucho', según el DFEM, p. 148), gastar saliva ('hablar inútilmente', según el DFEM, p. 251) o tirar con bala ('hablar con mala intención', según el DFEM, p. 20) son hipónimas del hiperónimo hablar, a la vez que mantienen entre sí una relación de cohiponimia. En ese estudio se analizan unas trescientas unidades fraseológicas del español equivalentes a sintagmas verbales, de modo que se da cuenta del alcance de la distinción género / especie en el ámbito de la fraseología y se explican los tipos de oposiciones entre el hipónimo y el hiperónimo. Por su parte, en I. Penadés Martínez (en prensa b) he aplicado los tipos de opuestos léxicos establecidos por J. Lyons (1993) a un conjunto de locuciones verbales del español para demostrar así la existencia de relaciones semánticas de oposición entre esa clase de unidades.

En el DICLOCVER se marcan las relaciones de sinonimia y antonimia entre dos locuciones remitiendo en la entrada de una locución a la entrada de la otra mediante el lema de esta última, y a la inversa. El lema de la locución sinónima a la que se remite va precedido de un símbolo que representa un puño cerrado con el dedo índice extendido señalando hacia la derecha; en el caso de ser antónimas las dos locuciones, el símbolo que precede al lema de ambas locuciones en una y otra entrada es el de una flecha orientada a la derecha. Cuando la locución sinónima o antónima de la definida tiene un lema idéntico al de otras locuciones, un exponente en negrita colocado en la parte superior derecha de la locución ( $\left.{ }^{1}\right)$ lo señala, y, además, la acepción que hay que considerar es la indicada por el exponente: 
dar carpetazo v. (III) [alguien, a algo] Desistir de una idea o de un proyecto: Hemos dado carpetazo a la construcción del edificio. dar marcha atrás ${ }^{2}$.

dar marcha atrás v. 1 (I) [algo/alguien] Retroceder: El conductor dio marcha atrás sin darse cuenta de que un niño cruzaba la calle; El coche dio marcha atrás. 2 (I) [alguien] Desistir de una idea o de un proyecto: Decidió dar marcha atrás y autorizar la emisión del anuncio. dar carpetazo.

Tanto la relación de sinonimia entre dos locuciones (marcada en sus respectivas entradas como se acaba de indicar), como la existente entre las locuciones de una serie sinonímica (marcada en las entradas como se ha explicado al presentar el apéndice de "Locuciones sinónimas y antónimas") se han establecido siempre que lo ha permitido la identidad en las definiciones de las locuciones ofrecidas por los distintos diccionarios que sustentan la elaboración de éste. Esa identidad ha sido en unos casos total, es decir, todos los diccionarios definen determinadas locuciones de la misma manera, o parcial, la coincidencia en la definición de dos o más locuciones sólo se da en algunos diccionarios.

Por otra parte, el establecimiento en el DICLOCVER de la relación semántica de sinonimia entre locuciones se ha llevado a cabo haciendo caso omiso de la distinción que pudiera existir entre el fenómeno de la variación léxica en el ámbito de la fraseología y el fenómeno de la sinonimia en el ámbito de la semántica. Quiere decirse con ello que locuciones como abrirse camino - abrirse paso, comerse un rosco-comerse una rosca o pedir guerra - querer guerra se presentan como sinónimas, igual que locuciones como echar chiribitas - estar que arde-subirse por las paredes o hacer caso omiso-pasarse por el forro (de los cojones) - saltar a la torera, aunque desde la perspectiva teórica de los fraseólogos aquéllas se consideren variantes léxicas ${ }^{31}$. La razón se encuentra, una vez más, en la orientación dada al diccionario. Pensando en los alumnos de E/LE, carece de sentido distinguir entre variación fraseológica y sinonimia si, al fin y al cabo, esa dicotomía no

31 Por ser modificaciones que tienen lugar en una misma construcción sintáctica, según Z. Carneado Moré (1985: 272). 
se traduce en un tratamiento lexicográfico diferenciado del significado de las locuciones que se consideren variantes léxicas frente a las que sean vistas como sinónimas. Además, el usuario del diccionario no hubiera comprendido que entre locuciones como retorcerse de risa, revolcarse de risa o tirarse de risa, variantes léxicas más que sinónimas desde el punto de vista que aquí estoy planteando, no se indicara la existencia de una relación de sinonimia, pues la identidad en la definición de las tres le da a entender a la persona que consulte el diccionario y que no sea especialista que tienen el mismo significado y que son sinónimas, no que son variantes léxicas de una misma locución verbal. El fenómeno lingüístico de la sinonimia entre dos o más unidades parece más cercano a la conciencia lingüística de los hablantes que no son estudiosos de las lenguas que el de la variación, sea ésta léxica, fonética o de cualquier otro tipo, pues reflexionar sobre la variación lingüística exige establecer cuál es la unidad básica, la invariante, de la que otra $u$ otras son variantes.

A nadie se le oculta la complejidad de las cuestiones referidas a la sinonimia como relación léxica, complejidad que se acrecienta si la perspectiva adoptada va más allá del ámbito del léxico y se adentra en la denominada sinonimia fraseológica. Dado el estado actual de las investigaciones sobre fraseología española, pero no sobre la fraseología teórica - pues de un tiempo a esta parte se han visto incrementadas en cuanto a su número y su calidad ${ }^{32}-$, sino sobre corpora que muestren el uso de las unidades fraseológicas, puede resultar extremadamente arriesgado ofrecer un tratamiento de la sinonimia fraseológica como el que se presenta en el DICLOCVER: se han vinculado mediante la relación de sinonimia locuciones verbales que podrían considerarse de distinta lengua funcional y que muestran combinatorias sintagmáticas diferentes. Sin embargo, y como queda dicho, estas circunstancias no han impedido señalar esa relación. Y ello, por una parte, por la creencia de que la elaboración de una obra de fraseografía aplicada (un diccionario de locuciones para estudiantes y profesores de E/LE) no puede pospo-

32 Vid., por ejemplo, G. Corpas (1996), L. Ruiz Gurillo (1997), J. de D. Luque y A. Pamies (1998) y G. Wotjak (1998). 
nerse hasta la resolución de determinados problemas por la fraseología teórica ${ }^{33} \mathrm{y}$, por otra parte, por el convencimiento de que el profesor de E/LE está perfectamente capacitado para hacerle notar al alumno las diferencias entre determinadas locuciones por su pertenencia a distintas diastratías o diafasías. No hay que olvidar tampoco que de esas diferencias se informa en distintas partes de la entrada de una locución.

La distancia entre teoría y aplicación en la investigación lingüística también ha condicionado la marcación de determinadas locuciones como antónimas. Esta relación léxica entre dos locuciones concretas se ha establecido, asimismo, en el DICLOCVER a partir de sus definiciones en los diccionarios que en todo momento se han consultado. Y, además, se ha tomado el término antonimia en un sentido amplio, de modo que bajo esa denominación quedan englobados tipos de opuestos que en J. Lyons (1993) se han diferenciado de los específicamente considerados antónimos. Junto a ello, no se han presentado como antónimas locuciones como alterar los nervios / alterarse los nervios o revolver el estómago / revolverse el estómago, que, desde la concepción de J. Lyons (1993), pueden analizarse como opuestos direccionales de consecuencia $^{34}$, pues, al fin y al cabo, se trataría, en este caso, no tanto de una oposición entre el significado léxico de las unidades fraseológicas de cada par, sino de una oposición diatética entre los esquemas oracionales cuyos núcleos son una y otra de las locuciones verbales de esos dos pares, relación de oposición compleja de percibir y entender por un usuario no especialista, sin las explicaciones pertinentes.

\subsection{LAS INDICACIONES GRAMATICALES Y PRAGMÁTICAS}

Finalmente, en el último apartado de los artículos de algunas entradas, introducido mediante un símbolo que representa un lápiz, se ofrecen, si es el caso, informaciones lingüísticas sobre diversas cuestiones relativas, fundamentalmente, a aspectos gramaticales y pragmáticos de

33 Piénsese que desde Aristóteles se viene reflexionando sobre la sinonimia y todavía no hemos dado con la solución al problema.

34 Vid. I. Penadés Martínez (en prensa b). 
la locución en la acepción definida, aunque, en alguna ocasión, la información ataña a aspectos ortográficos. Se ha aprovechado el apartado en cuestión para presentar aclaraciones sobre el contorno, sobre la posibilidad de variaciones morfológicas o sobre la fuerza ilocucional de las locuciones. Estas informaciones van acompañadas de ejemplos adicionales que las ilustran y que completan los incluidos en el apartado de la definición:

estar hasta los cataplines v. (a) infor. [alguien, de algo/alguien] Estar harto \{un hombre de una persona o de una cosa\}: Están hasta los cataplines de tus protestas. *estar hasta el gorro. El complemento [de algo] puede ser también un verbo en infinitivo o una oración subordinada introducida por de que: El vecino de abajo está hasta los cataplines de pintar su techo del cuarto de baño.

hacer picadillo v. (a) infor. [alguien, a alguien] Matar \{a una persona $\}$ : Los secuestradores dijeron a la policía que harían picadillo a los rehenes si no se cumplían sus condiciones. *borrar del mapa. La locución suele utilizarse como amenaza: Has vuelto a romper un cristal con la pelota, como te coja, te hago picadillo.

no ser santo de la/su devoción v. (s) infor. [alguien, de alguien] Resultar antipático a una persona: El primer ministro no es santo de la devoción ni de los miembros de la derecha ni de los de la izquierda de su propio partido. *aer bien. Si la forma de la locución es no ser santo de su devoción, la combinatoria de la locución es [alguien]: Cuando mi secretaria me dijo que él estaba personalmente al teléfono, me quedé atónito, pues yo nunca habia sido santo de su devoción, sino más bien todo lo contrario. El elemento su de la locución admite variación de $1^{\mathrm{a}}, 2^{\mathrm{a}}$ y $3^{\mathrm{a}}$ persona: He de admitir que no era santo de mi devoción, además de por lo ya dicho, porque era una persona tosca y vanidosa.

Y terminaré este artículo no con unas conclusiones, sino con un párrafo tomado de la "Presentación" del DICLOCVER, pues por la finalidad de esta publicación me parece más adecuado: "La adquisición del conocimiento científico por parte de la especie humana es siempre un logro limitado. Este diccionario, como no podía ser menos, se presenta como una obra abierta y sujeta a modificaciones y cambios, tanto más cuanto que tendrá su continuación en los volúmenes dedicados a las 
otras clases de locuciones. Por eso, los comentarios, las sugerencias y las observaciones críticas serán bien recibidos (en inmaculada.penades@uah.es)".

\section{REFERENCIAS BIBLIOGRÁFICAS}

Blanco CANAles, Ana (1997): Estudio sociolingüístico de la ciudad de Alcalá de Henares, Tesis doctoral mecanografiada, Universidad de Alcalá.

BRIZ, Antonio (coord.) (1995): La conversación coloquial. (Materiales para su estudio), Valencia, Universitat de València.

CARNEADO MoRÉ, Zoila (1985): "Notas sobre las variantes fraseológicas", Anuario L/L, 16, pp. 269-277.

CASARES, Julio (1969): Introducción a la lexicografía moderna, Madrid, C.S.I.C., reimp. [1950].

Cestero Mancera, Ana M ${ }^{\mathrm{a}}$. (2000): El intercambio de turno de habla en la conversación (análisis sociolingüistico), Alcalá de Henares, Servicio de Publicaciones de la Universidad de Alcalá.

Corpas PAstor, Gloria (1996): Manual de fraseología española, Madrid, Gredos.

Coseriu, Eugenio (1964): "Pour une sémantique diachronique structurale", Travaux de Linguistique et de Litérature, 2/, 1, pp. 139-186.

Forment FERNÁNDEZ, María del Mar (1998): "La didáctica de la fraseología ayer y hoy: del aprendizaje memorístico al agrupamiento en los repertorios de funciones comunicativas", en Francisco Moreno, María Gil y Kira Alonso (eds.), La enseñanza del español como lengua extranjera: del pasado al futuro. Actas del VIII Congreso Internacional de ASELE, Alcalá de Henares, Universidad de Alcalá, pp. 339-347.

GutiérRez CuAdrado, Juan (dir.) (1996): Diccionario Salamanca de la lengua española, Barcelona, Círculo de Lectores.

Kleiber, Georges e I. TAMBA (1990) : "L'hyponymie revisitée: inclusion et hiérarchie", Langages, 98, pp. 7-32.

Luque Durán, Juan de Dios y Antonio Pamies Bertrán (1998): Léxico y fraseología, Granada, Método Ediciones.

Lyons, John (1993): Semantics, 1, Cambridge, Cambridge University Press, $1^{\text {a }}$ ed., reimp. [1977]. 
Maldonado GonzÁlez, Concepción (dir.) (1994): Diccionario didáctico de español, intermedio, Madrid, Ediciones SM, $3^{\mathrm{a}}$. ed.

Moliner, María (1966-1967): Diccionario de uso del español, Madrid, Gredos.

Moon, Rosamund (1997): "Vocabulary connections: multi-word items in English", en Norbert Schmitt y Michael McCarthy (eds.), Vocabulary: description, acquisition and pedagogy, Cambridge, Cambridge University Press, pp. 40-63.

Moreno FernÁNDEZ, Francisco (coord.) (1995): Diccionario para la enseñanza de la lengua española, Alcalá de Henares / Barcelona, Universidad de Alcalá de Henares / Biblograf, $1^{\text {a }}$. ed.

Moreno Fernández, Francisco, Ana M ${ }^{\mathrm{a}}$ Cestero Mancera, Isabel MoLina Martos y Florentino Paredes García (2000): "La sociolingüística de Alcalá de Henares en el "Proyecto para el Estudio Sociolingüístico del Español de España y América" (PRESEEA)", Oralia, 3, pp. 149-168.

- (2002): La lengua hablada en Alcalá de Henares. Corpus PRESEEA - ALCALA. I. Hablantes de instrucción superior, Alcalá de Henares, Universidad de Alcalá.

PenAdés Martínez, Inmaculada (1999): La enseñanza de las unidades fraseológicas, Madrid, Arco/Libros.

(2000): La hiponima en las unidades fraseológicas, Cádiz, Universidad de Cádiz.

_- (2001): “¿Colocaciones o locuciones verbales?”, Lingü̈́stica Española Actual, XXIII/1, pp. 57-88.

—_ (2002): Diccionario de locuciones verbales para la enseñanza del español, Madrid, Arco/Libros.

(en prensa a): “¿Caracterizan las locuciones el registro coloquial?”, Actas del V Congreso de Lingüistica General, León, 5-8 de marzo de 2002.

(en prensa b): "Les relations d'opposition dans les locutions espagnoles", Cahiers de Lexicologie.

Real Academia Española (1992): Diccionario de la lengua española, Madrid, Espasa-Calpe, $21^{\text {a }}$. ed.

- (2001): Diccionario de la lengua española, Madrid, Espasa-Calpe, $22^{\mathrm{a}}$. ed.

RUIz GURILLO, Leonor (1997): Aspectos de fraseología teórica española, Valencia, Universitat de València.

Ruiz MartíneZ, Ana María (1999): Estudio lingüístico del nordeste de la 
Comunidad de Madrid, Tesis doctoral mecanografiada, Universidad de Alcalá.

_- (en prensa): Estudio fonético del nordeste de la Comunidad de Madrid, Alcalá de Henares, Universidad de Alcalá.

Seco, Manuel, Olimpia Andrés y Gabino Ramos (1999): Diccionario del español actual, Madrid, Aguilar, $1^{\text {a }}$. ed., $1^{\text {a }}$. reimp.

VARELA, Fernando y Hugo KUBARTH (1994): Diccionario fraseológico del español moderno, Madrid, Gredos.

WOTJAK, Gerd (1998): Estudios de fraseología y fraseografía del español actual, Frankfurt am Main / Madrid, Vervuert / Iberoamericana.

\section{ACRÓNIMOS DE LOS DICCIONARIOS}

DDEI: Diccionario didáctico de español intermedio, Concepción Maldonado González (dir.), Madrid, Ediciones SM, 1994.

DEA: Diccionario del español actual, Manuel Seco, Olimpia Andrés y Gabino Ramos, Madrid, Aguilar, 1999.

DFEM: Diccionario fraseológico del español moderno, Fernando Varela y Hugo Kubarth, Madrid, Gredos, 1994.

DICLOCVER: Diccionario de locuciones verbales para la enseñanza del español, Inmaculada Penadés Martínez, Madrid, Arco/Libros, 2002. DIPELE: Diccionario para la enseñanza de la lengua española, Francisco Moreno Fernández (coord.), Alcalá de Henares / Barcelona, Universidad de Alcalá de Henares / Biblograf, 1995.

DRAE: Diccionario de la lengua española, Real Academia Española, Madrid, Espasa-Calpe, 1992 y 2001.

DSLE: Diccionario Salamanca de la lengua española, Juan Gutiérrez Cuadrado (dir.), Barcelona, Círculo de Lectores, 1996.

DUE: Diccionario de uso del español, María Moliner, Madrid, Gredos, 1966-1967. 